\title{
New type of ensemble of quasi-periodic, long-lasting VLF emissions at the auroral zone
}

\author{
J. Manninen ${ }^{1}$, N. G. Kleimenova ${ }^{2,3}$, and O. V. Kozyreva ${ }^{2,3}$ \\ ${ }^{1}$ Sodankylä Geophysical Observatory, Finland \\ ${ }^{2}$ Institute of the Earth Physics RAS, Moscow, Russia \\ ${ }^{3}$ Space Research Institute, Moscow, Russia \\ Correspondence to: J. Manninen (jyrki.manninen@sgo.fi)
}

Received: 12 September 2012 - Accepted: 18 November 2012 - Published: 11 December 2012

\begin{abstract}
A new type of the series of quasi-periodic (QP) very low frequency (VLF) emissions in frequency range of $1-5 \mathrm{kHz}$, and not associated with geomagnetic pulsations, has been discovered at auroral latitudes $(L=5.3)$ during the Finnish VLF campaign (held in December 2011). At least five unusually spectacular events, each with a duration of several hours, have been observed during the night under conditions of quiet geomagnetic activity $(\mathrm{Kp}=0-1)$, although QPs usually occur during the daytime. Contrary to the QP emissions typically occurring during the day, the spectral structure of these QP events represented an extended, complicated sequence of repeated discrete rising VLF signals. Their duration was about 2-3 min each, with the repetition periods ranging from $\sim 1 \mathrm{~min}$ to $\sim 10 \mathrm{~min}$. Two such nighttime nontypical events are reported in this paper. The fine structure of the separated QP elements may represent a mixture of the different frequency band signals, which seem to have independent origins. It was found that the periodic signals with lower frequency appear to trigger the strong dispersive upper frequency signals. The temporal dynamics of the spectral structure of the QPs studied were significantly controlled by some disturbances in the solar wind and interplanetary magnetic field (IMF). This finding is very important for future theoretical investigations because the generation mechanism of this new type of QP emissions is not yet understood.
\end{abstract}

Keywords. Ionosphere (Wave propagation) - Magnetospheric physics (Magnetosphere-ionosphere interactions; Plasma waves and instabilities)

\section{Introduction}

Quasi-periodic (QP) very low frequency (VLF) emissions were first reported by Gallet (1959), Pope and Campbell (1960), and Lokken et al. (1961). Later, Helliwell (1965) divided these emissions into two classes: periodic emissions (Pe) represented a sequence of discrete events or clusters of discrete events showing regular spacing with typical periodicity in the range of $1-7 \mathrm{~s}$, and quasi-periodic emissions (QP) represented a sequence of repeated noise bursts of relatively long period ( $\sim 20-50 \mathrm{~s})$, in which each burst may consist of a number of discrete events or periodic emissions. The period between bursts is typically measured in tens of seconds. These emissions are understood to be whistler mode waves of magnetospheric origin that have propagated through the ionosphere to the ground.

QP emissions have subsequently been subdivided (Kitamura et al., 1968; Sato and Fukunishi, 1981) into two types: being either associated (QP1) or not associated (QP2) with geomagnetic pulsations. QP1 is more widely referred to in the literature (e.g., Kimura, 1974; Sato and Fukunishi, 1981; Bespalov and Kleimenova, 1989; Sazhin and Hayakawa, 1994; Manninen et al., 2012). However, there have been relatively few papers analysing QP2 emissions and described their behaviour (Ho, 1973; Morrison et al., 1994; Smith et al., 1998; Engebretson et al., 2004). Based on Antarctic observations, these authors concluded that QP2 are most common in austral summer under conditions of quiet geomagnetic activity, their diurnal maximum of occurrence is near noon, and the period of separated VLF bursts repetition ranged from $\sim 20 \mathrm{~s}$ to $\sim 100 \mathrm{~s}$. The QP emissions showed good conjugateness and the elements of QP emissions appeared 

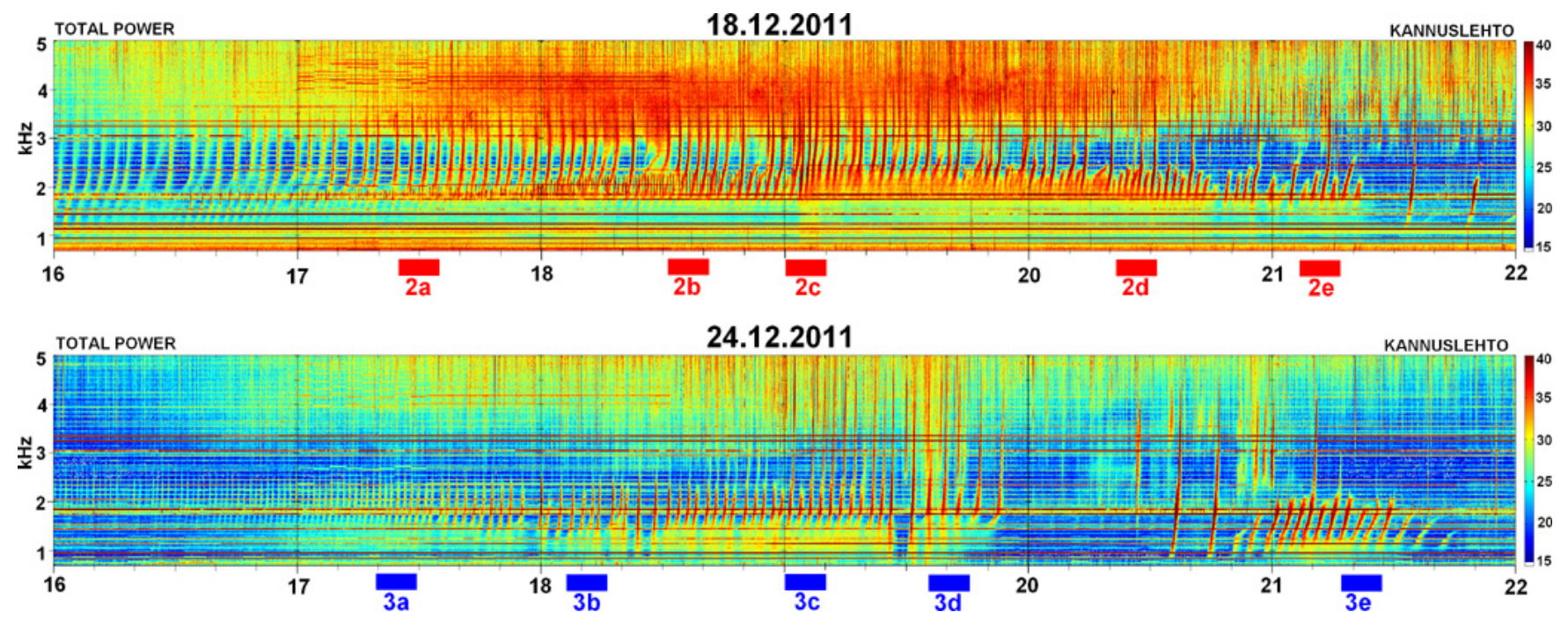

Fig. 1. Spectrograms showing two ensembles of QP emissions recorded in Northern Finland in December 2011.

simultaneously at the conjugate stations. Very often these VLF emissions were accompanied by periodic emissions with periods of a few seconds.

A new type of the series of QP $1-5 \mathrm{kHz}$ VLF emissions, not associated with geomagnetic pulsations, was noticed at $L \sim 5.3$ during the Finnish VLF campaign (held in December 2011). The occurrence of extended QPs, lasting 4-6h, and consisting of repeated, unusually long-lasting (up to two minutes) bursts of VLF emissions, at night, was perhaps surprising. The repetition period of the separate signals was large, and ranged from about one to about ten minutes. These events, not previously described in the literature, are the object of this paper.

\section{Observations}

Unusually long-lasting QP2 events were observed during the Finnish VLF campaign of December 2011 at a temporary station in Kannuslehto: $\varphi=67.74^{\circ} \mathrm{N}, \lambda=26.27^{\circ} \mathrm{E} ; L=5.3$, by applying a very high-sensitivity VLF receiver with the threshold on the order of $10^{-6} \mathrm{nT}$. At least 5 spectacular events, each lasting several hours, have been recorded under quiet geomagnetic activity $(\mathrm{Kp}=0-1)$. Most of them appeared in the late evening before local midnight or in the early morning after midnight. None of these QP emission events were associated with geomagnetic pulsations, so are classified as QP2 type.

Two examples of the unusual QP events recorded at nighttime, will be examined here - those from 18 and 24 December. The spectrograms of the QP events, each lasting about $6 \mathrm{~h}$, are given in Fig. 1, in the frequency range of $0.7-5.0 \mathrm{kHz}$. The strong horizontal lines in Fig. 1 are due to power line harmonic radiation (PLHR), which are multiples of $50 \mathrm{~Hz}$. Analysis of the wave polarisation showed that in both cases, the QP emissions were mostly right-hand polarised, indicating the location of the ionospheric VLF wave exit point was almost above the ground receiver. According to the model (http://www.spaceweather.eu/plasmapause), the plasmapause was located at $\mathrm{L} \sim 5$ during both events. Thus, we may suppose that the QP emissions studied here were generated in the vicinity of the plasmapause, and our VLF receiver might be located near the projection of the origin of these emissions.

\subsection{QP event on 18 December 2011}

The spectrograms of the QP emissions on 18 December 2011 are shown in the upper panel of Fig. 1. This event looks like a spectacular series of QP emissions with changing and complex spectral structure, decorated on top by a curtain of extended high-frequency $(\sim 3.0-4.5 \mathrm{kHz})$ hiss. The intensity and repetition frequency of QP emissions increased with the high-frequency hiss enhancement. The temporal dynamics of the spectral structure of the series of QPs under consideration are given in Fig. 2 as a sequence of five 10-min intervals on the spectrograms (shown in Fig. 1 by red bars).

It can be seen that at beginning of the event (Fig. 2a), the sparse individual long-lasting, rising, diffuse QP bursts at $\sim 1.8-3.5 \mathrm{kHz}$ were spaced $2-3 \mathrm{~min}$ apart, and accompanied by the rare lower-frequency narrow-band $(\sim 1.8-$ $2.0 \mathrm{kHz}$ ) short period rising signals, each of about $15 \mathrm{~s}$ duration. The appearance and generation of these two emissions with different frequency seems to be independent. With the increasing high-frequency hiss strength, the upper limit of the narrow-band emissions increased up to $2.3 \mathrm{kHz}$ (Fig. 2b). Around 19:00 UT, the low-frequency narrow-band emissions disappeared, but as seen in Fig. 2c, more probably the lower periodic seems to trigger the strong dispersive upper one. Later on (Fig. 2d), the high-frequency hiss broke up into 

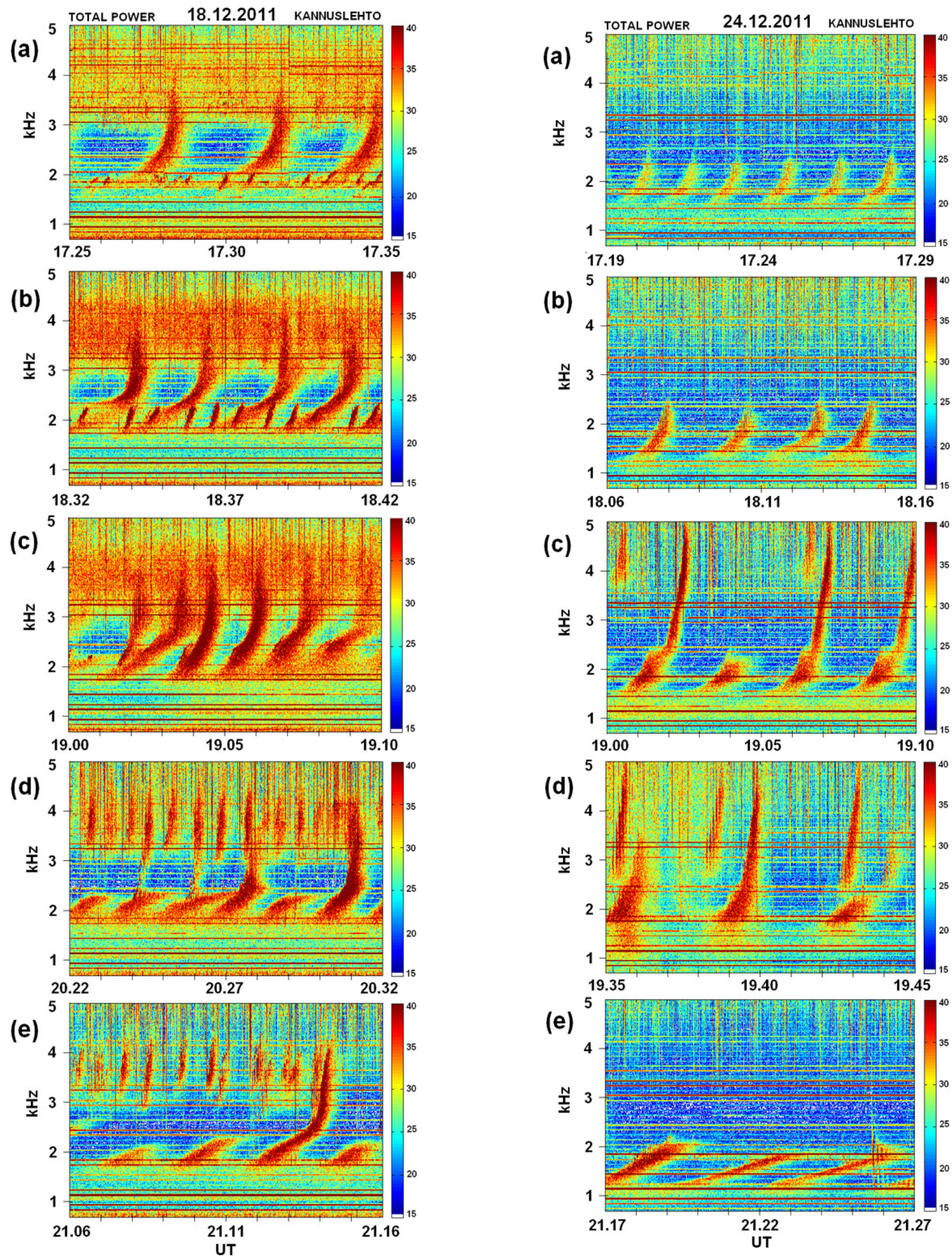

Fig. 2. Series of successive 10-min spectrograms of QP emissions on 18 December 2011.

Fig. 3. Series of successive 10-min spectrograms of QP emissions on 24 December 2011. 
(a)

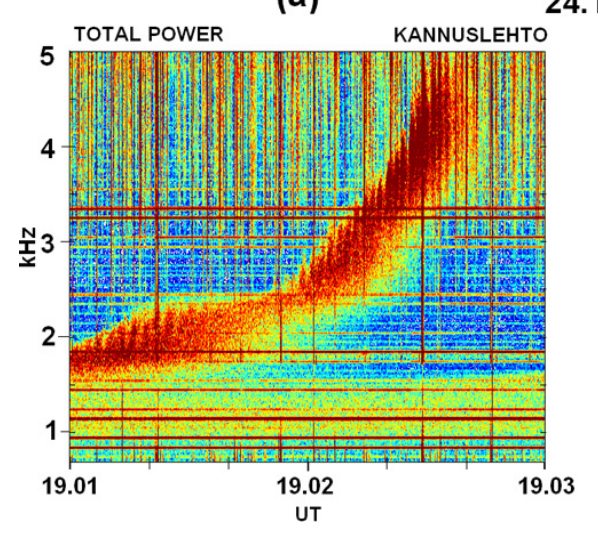

24.12.2011

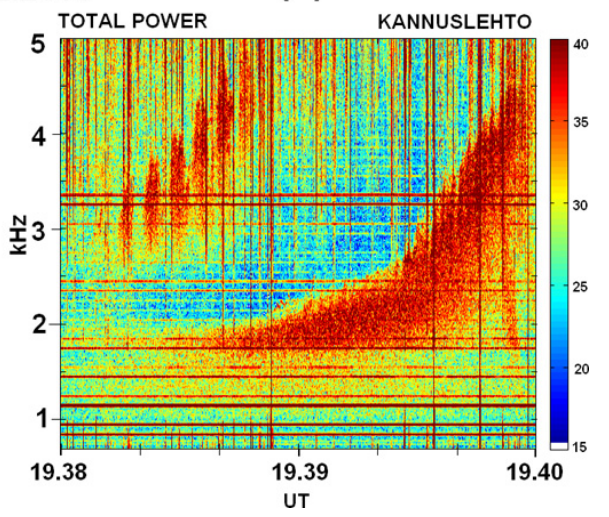

Fig. 4. Two 2-min spectrograms of selected QP emissions recorded during the event on 24 December 2011.

quasi-periodic noise patches with $\sim 40 \mathrm{~s}$ repetition. The lowfrequency narrow-band emissions appeared again as separated rising diffuse bursts with $\sim 1-2$ min periodicity, its upper frequency boundary became modulated by short $(\sim 6-$ $7 \mathrm{~s}$ ) periodic emissions. Some of the lower frequency narrowband emissions appear to trigger the upper ones as well as in preceded time. The event discussed here ended with these 2min repeated low-frequency, narrow-band, slow-rising emissions (Fig. 2e).

\subsection{QP event on 24 December 2011}

The spectacular event on 24 December 2011 was observed on Christmas night. The spectrogram of this event is presented in the bottom panel of Fig. 1. The signal dynamic spectra of 10-min intervals are given in Fig. 3 and shown by the blue bars in Fig. 1. The high-frequency hiss was observed on 18 December was also evident, but with much less strength. The event started with a series of the quasi-regular, diffuse, rising bursts in the frequency range of $\sim 1.7-2.4 \mathrm{kHz}$ and with repetition periods of $\sim 1.0-1.5 \mathrm{~min}$ (Fig. 3a).

Around 18:00 UT, the intensity of the bursts increased, and the lower frequency limit shifted down to $\sim 1.2 \mathrm{kHz}$ (Fig. 3b). Also, modulation with the period of a few seconds occurred near the top of every burst. The spectral structure of QP emissions changed after 19:00 UT (Fig. 3c): some of the periodic emissions at lower frequencies appear to trigger the strong dispersive tail up to $5 \mathrm{kHz}$. An example 2-min spectrogram of signal like this is given in Fig. 4a. This shows that the high frequency boundary of emission burst consists of periodic emissions with 6-7 s repetition periods. About 30 min later, a new modification of the QP structure was observed (Fig. 3d). The 2-min spectrogram (Fig. 4b) shows new $\sim 2.7-5.0 \mathrm{kHz}$ hiss patches (19:38-19:39 UT), consisting of a cluster which united several 4-min modulated noise bursts. This modulation was not seen in the next long-lasting QP element at a similar frequency. These emissions might have different sources. Similarly to the 18 December 2011 event, this event ended with repeated, low-frequency $(\sim 1-2 \mathrm{kHz})$ narrow-band, slow-rising emissions (Fig. 3e).

\section{Discussion}

In spite of the quiet geomagnetic conditions $(K \sim 0-1)$, some disturbances in the interplanetary magnetic field (IMF) and in the solar wind took place during both the QP events discussed as is shown in the upper parts of Fig. 5a and b (1-min OMNI data). During the first QP event on 18 December 2011 (Fig. 5a), a storm sudden commencement (SSC) occurred at 19:03 UT. This caused a strong jump in the solar wind density and velocity at $\sim 19.15 \mathrm{UT}$; and before that, a small, short negative excursion of IMF $B_{\mathrm{Z}}$ caused an enhancement of the AE-index and a corresponding small substorm (bottom part of Fig. 5a). A change in the QP dynamic spectra is seen at that time (Fig. 2c). A noticeable negative magnetic transient appeared in the IMF (both in IMF $B_{\mathrm{X}}$ and IMF $B_{\mathrm{Z}}$ ) at $\sim 20: 45 \mathrm{UT}$. Around this time, the high-frequency hiss practically disappeared, and the QP spectra experienced modification. Thus, it seems that IMF and solar wind state have significant influence on the spectral dynamics of QP emissions.

During the second QP event (24 December 2011), a small jump of the solar wind density and velocity took place at $\sim 18: 00$ UT (Fig. 5b), although it was much smaller than on 18 December 2011. However, after this, the low frequency limit of QPs shifted down to $\sim 1.2 \mathrm{kHz}$ (Fig. 3b). At 19:00 UT the IMF $B_{\mathrm{Z}}$ turned to the negative values, and the dynamic spectra of the QPs also changed. Furthermore, some interaction between the lower and higher frequency parts of QP bursts appeared (Fig. 3c). The other significant modification of the QP dynamic spectra was observed near 19:30 UT (Fig. 3d). This coincided with a relatively strong substorm with AE-index increasing up to $\sim 280 \mathrm{nT}$, which was recorded only at high latitudes (BJN, HOR). There were no substorms between 20:00 UT and 21:00 UT, but during 

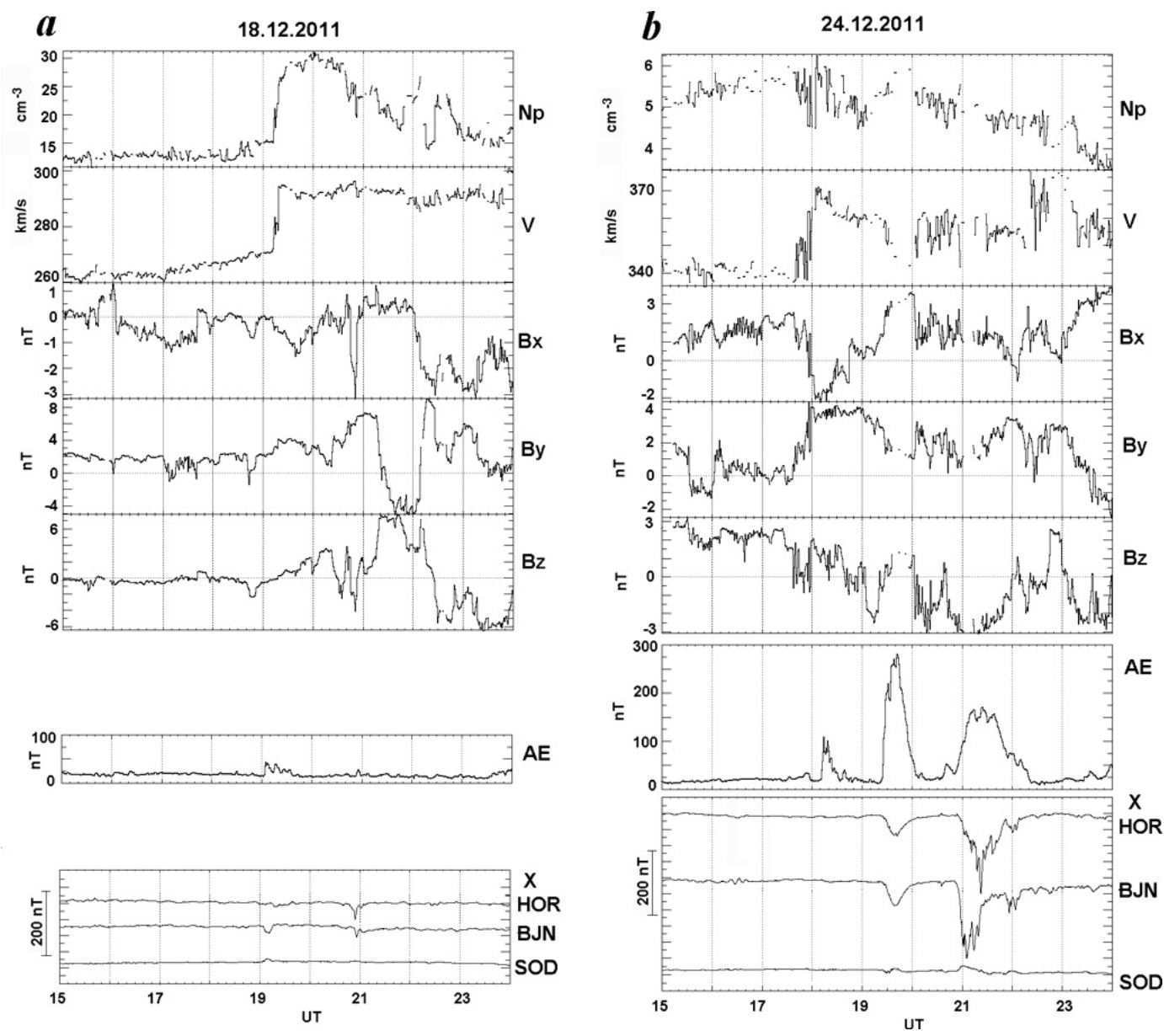

Fig. 5. OMNI data of solar wind density $\left(N_{\mathrm{p}}\right)$, velocity $(V)$ and interplanetary magnetic field (IMF) $B_{\mathrm{X}}, B_{\mathrm{y}}$, and $B_{\mathrm{Z}}$ components; AE-index

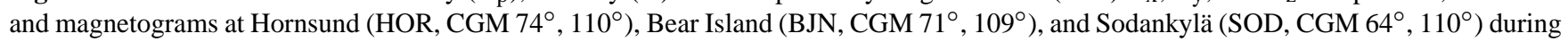
the event on (a) 18 December 2011, and (b) 24 December 2011.

this period both $3-5 \mathrm{kHz}$ hiss and QPs disappeared. No reasonable explanation has been found for this so far, and that will be one of the potential questions for future theoretical investigation. A new substorm developed near 21:00 UT, and at this time QP emissions appeared again.

Since this was the first time such a spectacular ensemble of different QP emissions has been observed, it is unsurprising that the generation mechanism and source of QPs described above is unknown. According to the theory of the cyclotron instability in the magnetospheric plasma maser (Trakhtengerts and Rycroft, 2008), one plausible reason for generation of QP emissions might be the auto-oscillation of the Earth's radiation belt electrons (Bespalov, 1982; Bespalov and Trakhtengerts, 1986).

\section{Summary}

A new type of ensemble of different quasi-periodic (QP) VLF emissions have been recorded in Northern Finland at
$L \sim 5.3$ during the Finnish VLF campaign held in December 2011. They appeared in the frequency range of $1-5 \mathrm{kHz}$. Unlike typical QP emissions, they were not associated with geomagnetic pulsations, and their spectral structure represented a sequence of repeated discrete and diffuse rising VLF signals, lasting about 2-3 min, with repetition periods ranging from about 1 to $10 \mathrm{~min}$. The emissions were observed at night, while typical QPs occur during the day.

It was found that fine structure of the separated QP elements may represent a mixture of different frequency bands signals, which seem to be of independent origins. In addition, some of the periodic emissions at lower frequencies appear to trigger a strong dispersive tail up to $5 \mathrm{kHz}$.

The temporal dynamics of the spectral structure of QPs were significantly influenced by some disturbances in the solar wind and IMF. This finding is very important for future theoretical investigations because the generation mechanism of the revealed type of QP emissions is not yet understood. 
Acknowledgements. The authors are very grateful to Tauno Turunen for special analysis programs and continuous valuable discussions and help during the Finnish VLF campaigns. N.K. and O.K. acknowledge Sodankylä Geophysical Observatory for support during their stay in Sodankylä, their work was partly supported by RFBR grants No. 10-05-00247 and 12-05-01030, and Program RAS No 22. The authors acknowledge the data provider J. H. King and N. Papitashvilli at NASA GSFC and CDAWeb for the ONMI data. We also thank Sally Ulich who checked the language.

Topical Editor K. Kauristie thanks M. J. Rycroft for his help in evaluating this paper.

\section{References}

Bespalov, P. A.: Self-excitation of periodic cyclotron instability regimes in a plasma magnetic trap, Physica Scripta, 2/2, 575579, 1982.

Bespalov, P. A. and Kleimenova, N. G.: Effect of geomagnetic pulsations on whistlers near the plasmapause, Geomagn. and Aeron., 29, 177-191, 1989.

Bespalov, P. A. and Trakhtengerts, V. Yu.: The cyclotron instability in the Earth radiation belts, in: Reviews of Plasma Physics, edited by: Leontovich, M. A., Plenum Publ., N.Y., 10, 155-292, 1986.

Engebretson, M. J., Posch, J. L., Halford, A. J., Shelburne, G. A., Smith, A. J., Spasojevic, M., Inan, U. S., and Arnoldy, R. L.: Latitudinal and seasonal variations of quasiperiodic and periodic VLF emissions in the outer magnetosphere, J. Geophys. Res., 109, A05216, doi:10.1029/2003JA010335, 2004.

Gallet, R. M.: The low frequency emissions generated in the Earth's exosphere, Proc. IRE, 47, 211-231, 1959.

Helliwell, R. A.: Whistler and related ionospheric phenomena, Stanford University Press, Stanford, California, 1965.
Ho, D.: Interactions between whistlers and quasi-periodic VLF emissions, J. Geophys. Res., 78, 7347-7356, 1973.

Kimura, I.: Interrelation between VLF and ULF emissions, Space Sci. Rev., 16, 389-411, 1974.

Kitamura, T., Jacobs, J. A., Watanabe, T., and Flint Jr., R. B.: Investigation of quasi-periodic VLF emissions and their relation to geomagnetic micropulsations, Nature, 220, 360-361, 1968.

Lokken, J. E., Shand, J. A., Wright, C. S., Martin, L. H., Brice, N. M., and Helliwell, R. A.: Stanford - Pacific Naval Laboratory conjugate point experiment, Nature, 192, 319-321, 1961.

Manninen, J., Kleimenova, N. G., Kozyreva, O. V., Bespalov, P. A., and Raita, T.: Quasi-periodic VLF emissions, VLF chorus and geomagnetic Pc4 pulsations (event on April 3, 2011), Geomagn. Aeron., 52, 82-92, 2012.

Morrison, K., Engebretson, M. J., Beck, J. R., Johnson, J. E., Arnoldy, R. L., Cahill Jr., L. J., Carpenter, D. L., and Gallani, M.: A study of quasi-periodic ELF-VLF emissions at three Antarctic stations: evidence for off-equatorial generation?, Ann. Geophys., 12, 139-146, doi:10.1007/s00585-994-0139-8, 1994.

Pope, J. H. and Campbell, W. H.: Observation of a unique VLF emissions, J. Geophys. Res., 65, 2543-2544, 1960.

Sato, N. and Fukunishi, H.: Interaction between ELF-VLF emissions and magnetic pulsations: Classification of quasi-periodic ELF-VLF emissions based on frequency-time spectra, J. Geophys. Res., 86, 19-29, 1981.

Sazhin, S. S. and Hayakawa, M.: Periodic and quasiperiodic VLF emissions, J. Atmos. Terr. Phys., 56, 735-753, 1994.

Smith, A. J., Engebretson, M. J., Klatt, E. M., Inan, U. S., Arnoldy, R. L., and Fukunishi, H.: Periodic and quasiperiodic ELF/VLF emissions observed by an array of Antarctic stations, J. Geophys. Res., 103, 23611-23622, 1998.

Trakhtengerts, V. Y. and Rycroft, M. J.: Whistler and Alfven mode cyclotron masers in space, Cambridge University Press, pp. 354, 2008. 